\title{
Organización del trabajo familiar y fecundidad en el México rural
}

\author{
María Elena Zúñiga, Carlos Santos, \\ Catherine Menkes \\ y Daniel Hernández*
}

\begin{abstract}
En este trabajo se analiza la organización económica familiar y su relación con la fecundidad y el uso de métodos anticonceptivos en cuatro grupos sociales del México rural. La información proviene de la Encuesta Rural de Planificación Familiar de 1981. Los cuatro grupos son: productores y asalariados agrícolas, trabajadores por cuenta propia y asalariados no agrícolas. Asimismo, para un segundo análisis de los productores, se les divide según su nivel de tecnificación.

En el conjunto de la población rural, el nivel de participación económica de las personas mayores de 7 años es superior a $50 \%$, siendo más elevada en los grupos que conforman unidades domésticas de producción (grupos no asalariados); sin embargo, éstos no son necesariamente los grupos donde se observan los mayores niveles de fecundiad. Por lo tanto, los autores plantean que la explicación de la desigual conducta reproductiva encontrada debe buscarse en la peculiar inserción de cada grupo social en los mercados de bienes, trabajo y dinero.
\end{abstract}

\section{Introducción ${ }^{1}$}

El problema que nos hemos planteado analizar en este trabajo es el de la importancia de la fuerza de trabajo familiar para la reproducción y sobrevivencia de la familia campesina y su relación con su conducta reproductiva. En este sentido, el análisis se centrará en la población que depende para su reproducción principalmente de la explotación de una parcela, a la que hemos denominado productores agrícolas. Como veremos más adelante, dicho grupo está conformado mayoritariamente por población campesina. Incluimos además en el análisis otros grupos sociales presentes en las localidades menores de 2500 habitantes. Su comparación con Social.

* Jefatura de Servicios de Planificación Familiar, Instituto Mexicano del Seguro

${ }^{1}$ Este trabajo presenta algunos de los resultados de la investigación "Análisis de la conducta reproductiva de los grupos sociales que conforman la estructura social en las áreas rurales de México, 1981" , iniciada en enero de 1983 bajo el patrocinio y apoyo de la Jefatura de Servicios de Planificación Familiar del Instituto Mexicano del Seguro Social, del Programa de Investigación Social en Población en América Latina y de la Academía Mexicana de Investigación en Demografía Médica. La información proviene de la "Encuesta Rural de Planificación Familiar, 1981", aplicada en 8049 hogares en 422 localidades de menos de 2500 habitantes, cuya realización estuvo a cargo de la mencionada Jefatura del IMSS. (IMSS-ISPF, 1982). 
el grupo de productores agrícolas, en cuanto al uso y destino de la fuerza de trabajo familiar, nos permitirá encontrar la especificidad de la organización y división del trabajo de las familias campesinas.

Para la construcción de los grupos sociales se tomó como base la ocupación principal del jefe económico (quien aporta más ingreso para el sostenimiento del hogar). Los factores centrales de la clasificación fueron la realización o no de una actividad agrícola y trabajar por un salario o en forma independiente. Los grupos que se definieron de esa manera son entonces: los productores agrícolas, los asalariados agrícolas, los trabajadores por cuenta propia y los asalariados no agrícolas.

Decíamos que los productores agrícolas podían ilustrar el comportamiento de la población campesina que reside en localidades menores de 2500 habitantes, debido a que las características que presentan son propias de este tipo de productores. Al campesino lo concebimos como un productor directo que no está separado de su medio de producción básico, la tierra, y que produce en condiciones no capitalistas, esto es, que el fin último de su proceso de producción no es un proceso de valorización de capital. Esto es resultado de su escasa dotación de medios de producción, por lo que estructuralmente su capacidad productiva es limitada (véase Bartra, 1979, pp. 85 y ss.).

Para determinar si un productor agrícola es campesino consideramos como principal criterio la capacidad de acumulación. En la medida que este indicador requiere para su captación de un seguimiento detallado y a profundidad, hemos utilizado los estudios de la CEPAL (véase CEPAL, 1982, pp. 104-109) para establecer un criterio cuantificable que nos aproxime a esta definición. Para lograr la sobrevivencia de una familia campesina promedio (5.5 personas) la CEPAL establece como mínimo una parcela de 8 hectáreas de equivalente de temporal nacional (ETN); para obtener un producto de reposición de la unidad de producción se requiere un mínimo de 12 hectáreas ETN. Una parcela mayor de 12 hectáreas permite obtener un producto excedente y con una parceỉa menor de 5 hectáreas no se obtiene siquiera el producto necesario para satisfacer los requerimientos básicos de una familia.

Siguiendo este criterio y haciendo las conversiones correspondientes a cada estado del país en hectáreas de ETN, encontramos que el grupo de productores agrícolas de la muestra está constituido en $75 \%$ por productores con menos de 5 hectáreas ETN, $88.7 \%$ por productores con menos de 9 hectáreas y $92.8 \%$ con menos de 12 hectáreas. De manera que sólo $7.2 \%$ del grupo de productores agrícolas tiene, en términos generales, cierta capacidad para obtener un producto excedente. Como es sabido, éste no siempre permite o no se utiliza para llevar a cabo un proceso de acumulación capitalista, por lo que no podemos saber cuántos de los productores sean capitalistas propiamente dichos. Sin embargo, podemos afirmar que, puesto que casi $93 \%$ de estos productores no encuentra en la parcela con- 
diciones para la obtención siquiera de un producto excedente, el grupo está conformado esencialmente por población campesina.

Considerando al conjunto de la población de jefes económicos, el grupo de productores agrícolas concentra a la mitad (51.5\%). Un $15 \%$ adicional de los jefes lo constituyen los asalariados agrícolas, otro $21.4 \%$ los asalariados no agrícolas $\mathrm{y}$, por último, los jefes trabajadores por cuenta propia conforman el $12.1 \%$ restante.

\section{Uso y división de la fuerza de trabajo familiar}

La importancia de estudiar el uso y destino de la fuerza de trabajo en la población campesina se desprende del vínculo peculiar que se presenta en estas unidades familiares entre la producción económica y la reproducción de la fuerza de trabajo. La unidad familiar campesina es una unidad económica, lo que le imprime necesidades y posibilidades específicas de funcionamiento (Martínez y Rendón, 1982, pp. 601-603).

Las relaciones sociales de producción predominantes para lograr la reproducción de la unidad económica campesina no son retribuidas monetariamente; en ellas participan los miembros de la familia, quienes de esta manera permiten la reproducción de la unidad económica, es decir, posibilitan la permanencia de su condición campesina. Esto es resultado de las características propias del proceso de producción campesina y, esencialmente, de su condición de subsistencia (Martínez y Rendón, 1982, p. 601; Warman, 1976, pp. 310-312; Martínez y Rendón, 1978, pp. 665-666). En ésta, el uso de fuerza de trabajo familiar tiene una gran importancia pues el productor difícilmente podría mantenerse contratando exclusivamente fuerza de trabajo al valor socialmente necesario para su reproducción (Martínez y Rendón, 1978, citado en Margulis, 1979). El productor tiene que hacer uso de la fuerza de trabajo con que cuenta, la suya y la de su propia familia, evitando de esta manera el pago de salarios durante gran parte de o todo el ciclo de cultivo. Así, para la producción campesina es de gran importancia contar con fuerza de trabajo familiar, la que a su vez depende del producto parcelario para reproducirse. Esta interrelación y dependencia de las esferas de la reproducción y de la producción. da lugar a una organización familiar basada en el uso de fuerza de trabajo familiar para la realización de las tareas productivas.

Para el análisis del uso de la fuerza de trabajo se estableció que los individuos en edad de trabajar eran todos aquellos de 7 años y más. El primer aspecto que resalta es que más de la mitad de la población en edad de trabajar de las localidades menores de $2 \mathbf{5 0 0}$ habitantes realiza una actividad económica (véase el cuadro 1). Esta proporción varía en los diferentes grupos sociales, siendo mayor la participación económica en el caso de los productores agrícolas y en el grupo de trabajadores por cuenta 
propia. Los niveles de participación tan altos y semejantes, presentes en ambos grupos, sugieren que el trabajo no asalariado se asocia a una mayor utilización de la fuerza de trabajo familiar. Por el contrario, los grupos en que la principal fuente de ingreso es la venta de fuerza de trabajo, es decir aquellos involucrados fundamentalmente en relaciones de producción típicamente capitalistas, presentan la menor proporción de personas ocupadas.

Las diferencias se acentúan cuarido se observa el nivel de participación de la población en los distintos grupos de edad y sexo. Así, mientras que entre los asalariados no agrícolas los niños menores de 16 años participan económicamente en $19.1 \%$, entre los campesinos esta proporción alcanza $57.4 \%$ (véase el cuadro 1). En general, la participación en actividades productivas por parte del sexo femenino es inferior a la que presentan los hombres en todas las edades y en cualquier grupo social, aunque la excepción la constituye el grupo de trabajadores por cuenta propia. Éste presenta peculiaridades en cuanto a los hombres y mujeres miembros de la familia que trabajan. En el grupo de trabajadores por cuenta propia, la participación de las mujeres de cualquier edad es significativamente mayor que la que presentan los otros grupos. Entre aquellas de 61 años y más trabajan casi 7 de cada 10, mientras que los campesinos muestran en este aspecto un porcentaje de alrededor de 23 .

CUADRO 1

Proporción de personas mayores de 7 años que trabajan en cada grupo social según sexo y tres grupos de edad

\begin{tabular}{|c|c|c|c|c|c|c|c|}
\hline \multirow[b]{2}{*}{ Grupos sociales } & \multicolumn{3}{|c|}{ Hombres } & \multicolumn{3}{|c|}{ Mujeres } & \multirow[b]{2}{*}{ Total } \\
\hline & 8-15 & $16-60$ & 61 y más & $8-15$ & $16-60$ & 61 y más & \\
\hline $\begin{array}{l}\text { Productores } \\
\text { agricolas } \\
\text { Asalariados }\end{array}$ & 57.4 & 96.0 & 90.0 & 25.9 & 39.0 & 23.5 & 59.1 \\
\hline agrícolas & 28.9 & 96.5 & 81.8 & 12.6 & 23.5 & 19.8 & 47.3 \\
\hline $\begin{array}{l}\text { Cuenta propia } \\
\text { Asalariados }\end{array}$ & 45.9 & 91.2 & 81.5 & 27.4 & 54.1 & 65.6 & 59.3 \\
\hline no agrícolas & 19.1 & 92.3 & 60.0 & 9.7 & 23.0 & 17.4 & 44.1 \\
\hline Total & 45.3 & 94.9 & 85.2 & 21.2 & 35.4 & 30.1 & 54.6 \\
\hline
\end{tabular}

La fuerte participación femenina en este grupo social, como veremos más adelante, está asociada a las características del recurso principal con que cuenta el grupo de trabajadores por cuenta propia. El negocio familiar permite el empleo de las mujeres, sobre todo adultas, ya que es más compatible con las labores domésticas, pues en la mayoría de los casos 
se realiza dentro del propio hogar. Por otro lado, ante la falta de este tipo de recursos, el empleo de la fuerza de trabajo femenina depende de las características de los mercados locales de trabajo en los que, en la mayoría de los casos, existe una preferencia por trabajadores masculinos adultos. Esto puede estar influyendo en los niveles tan bajos de participación económica de las mujeres de todas las edades en los otros grupos sociales, especialmente en los asalariados.

En cierta forma, algo parecido puede estar ocurriendo con los hombres mayores de 60 años. En el grupo de productores agrícolas, $90.0 \%$ del total participa en procesos productivos; esta proporción es de alrededor de 81 para los grupos de asalariados agrícolas y trabajadores por cuenta propia, y de 60 para los asalariados no agrícolas. Se puede pensar que la fuerza de trabajo de los hombres en estas edades encuentra mayores posibilidades de ocupación cuando se cuenta con un recurso productivo propio, sobre todo la parcela, donde además puede significar una descarga para aquellos miembros que tienen mejores oportunidades de ocupación en los mercados de trabajo locales y foráneos. Por otro lado, las condiciones de vida de los asalariados agrícolas están requiriendo que los trabajadores, sobre todo masculinos, continúen trabajando aún a edades muy avanzadas.

Resumiendo, los grupos de productores agrícolas y trabajadores por cuenta propia son los que presentan la mayor participación económica de los integrantes de sus unidades debido a los niveles tan altos en el uso de la fuerza de trabajo de niños, mujeres y ancianos. Los grupos de asalariados, por el contrario, son los que presentan una menor proporción de trabajadores totales. En el caso de los asalariados agrícolas, el nivel de participación está caracterizado por el menor uso de fuerza de trabajo infantil y femenina, aunque ésta es, en la gran mayoría de los casos, utilizada en mayor proporción que en el grupo de asalariados no agrícolas. Éste es el grupo que muestra, con mucho, el más bajo nivel de participación económica del conjunto de sus integrantes, responsabilidad que recae esencialmente en los trabajadores masculinos de 16 a 60 años (véase el cuadro 1).

Pasaremos ahora a ver cuál es el comportamiento de los grupos sociales respecto a la diversificación de actividades, tanto de un mismo individuo como de los integrantes de cada grupo social en las distintas actividades económicas.

$\mathrm{Al}$ considerar el número de personas que realizan más de una actividad, se observa cómo los grupos independientes, ya sea de productores agrícolas o los trabajadores por cuenta propia, son los que presentan la mayor proporción de individuos que realizan más de un trabajo, sin mayores diferencias entre ellos (véase el cuadro 2). La misma relación se presenta cuando se analiza el número de actividades distintas que realiza el conjunto de los miembros de cada grupo. La posibilidad de desarrollar una actividad económica propia o independiente tiene una importante re- 
CUADRO 2

Porcentaje de trabajadores respecto al total de población ocupada que realizan más de una actividad económica en cada grupo social

\begin{tabular}{lc}
\hline Productores agrícolas & 22.2 \\
Asalariados agrícolas & 14.3 \\
Cuenta propia & 22.5 \\
Asalariados no agrícolas & 10.9 \\
\hline
\end{tabular}

Fuente: Encuesta Rural de Planificación Familiar (1981).

percusión sobre la diversificación del uso del trabajo familiar, esto es, amplía el margen de decisión y "control" sobre el empleo de los integrantes del hogar, lo que no sucede en el caso de los grupos asalariados.

Se puede ver que en la actividad principal del grupo, esto es, la parcela para los productores agrícolas, el negocio familiar para el grupo de trabajadores por cuenta propia y la venta de fuerza de trabajo para los grupos de asalariados, concentra el $68.5,59.5,73$ y 76.5 por ciento del total de personas que trabajan, respectivamente (véase el cuadro 3 ).

CUADRO 3

Distribución porcentual de los individuos que trabajan según actividad que realizan y grupo social

\begin{tabular}{|c|c|c|c|c|c|}
\hline & $\begin{array}{c}\text { Productores } \\
\text { agrícolas }\end{array}$ & $\begin{array}{c}\text { Asalariados } \\
\text { agrícolas }\end{array}$ & $\begin{array}{l}\text { Cuenta } \\
\text { propia }\end{array}$ & $\begin{array}{l}\text { Asalariados } \\
\text { no agrícolas }\end{array}$ & Total \\
\hline Sólo parcela & 68.5 & 9.6 & 5.0 & 7.1 & 44.3 \\
\hline Parcela y VFT* & 14.5 & 11.7 & 0.6 & 7.9 & 11.4 \\
\hline Sólo VFT & 4.2 & 73.0 & 9.5 & 76.5 & 24.3 \\
\hline Sólo negocio & 4.9 & 3.4 & 59.5 & 5.1 & 11.3 \\
\hline Parcela y negocio & 6.0 & 0.3 & 14.0 & 0.6 & 5.4 \\
\hline Otras combinaciones & 1.9 & 2.1 & 11.5 & 2.7 & 3.2 \\
\hline Total & 100.0 & 100.0 & 100.0 & 100.0 & 100.0 \\
\hline VFT sólo y/u otro & 19.8 & 86.2 & 14.9 & 86.8 & 37.5 \\
\hline Parcela sólo $\mathrm{y} / \mathrm{u}$ otro & 90.4 & 21.7 & 22.3 & 16.1 & 62.4 \\
\hline Negocio sólo $\mathrm{y} / \mathrm{u}$ otro & 12.1 & 5.6 & 80.9 & 8.1 & 19.0 \\
\hline
\end{tabular}

La mayor diversificación de actividades en el grupo de trabajadores por cuenta propia respecto al de productores agrícolas se puede explicar por el hecho de que los primeros cuentan con más recursos propios (véase el cuadro 4). Una quinta parte de los trabajadores por cuenta propia puede incluso emplearse en una parcela del hogar, al menos parcialmente, mientras que, a la inversa, entre los productores agrícolas la ocupación en un negocio familiar sólo la presenta $12 \%$ del total de trabajadores 
de manera sola o combinada con otras actividades (parte inferior del cuadro 3). Sin embargo, puede verse la importancia que tiene para los productores agrícolas la venta de fuerza de trabajo, pues uno de cada cinco trabajadores recibe un salario en al menos una de las actividades que realiza. Esto puede estar reflejando la necesidad de obtener un ingreso monetario que complemente el presupuesto familiar.

CUADRO 4

Distribución porcentual de los hogares de los distintos grupos sociales según los recursos con los que cuentan

\begin{tabular}{lcccc}
\hline & $\begin{array}{c}\text { Productores } \\
\text { agrícolas }\end{array}$ & $\begin{array}{c}\text { Asalariados } \\
\text { agrícolas }\end{array}$ & $\begin{array}{c}\text { Trabajadores } \\
\text { por cuenta } \\
\text { propia }\end{array}$ & $\begin{array}{c}\text { Asalariados } \\
\text { no agrícolas }\end{array}$ \\
\hline $\begin{array}{l}\text { Sólo parcela } \\
\text { Sólo negocio }\end{array}$ & 84.0 & 16.2 & 0.0 & 10.4 \\
$\begin{array}{c}\text { Parcela y ne- } \\
\text { gocio }\end{array}$ & 0.0 & 6.0 & 74.0 & 5.4 \\
$\begin{array}{c}\text { Sin parcela ni } \\
\text { negocio }\end{array}$ & 16.0 & 1.2 & 26.0 & 2.6 \\
\hline
\end{tabular}

Fuente: Encuesta Rural de Planificación Familiar (1981).

Entre los asalariados agrícolas se puede presentar el caso inverso, pues la parcela constituye, en ciertos periodos, la única fuente de ingresos con que se cuenta, dada la irregularidad en el empleo en el mercado laboral agrícola. De aquí el esfuerzo realizado por los asalariados que tienen parcela por mantener su vínculo con la tierra, pese a que la venta de fuerza de trabajo les aporte la mayor parte de los ingresos que requieren para su reproducción (véase el cuadro 4). Esto también puede explicar el mayor uso de trabajadores marginales que veíamos al principio de este apartado.

A partir de los datos analizados hasta aquí se observa cómo en los grupos de trabajadores independientes las personas tienen una mayor participación económica y diversifican en mayor medida el uso de su fuerza de trabajo. Asimismo, hemos resaltado cómo el empleo de los miembros del hogar tiene diferente importancia, dependiendo de la edad y sexo de las personas en los diferentes grupos sociales. Resta por considerar de qué manera se asignan las diferentes labores productivas a cada tipo de trabajador. Para el análisis de la división del trabajo hemos clasificado a los trabajadores, según edades y sexo, en tres tipos: marginales (de 8 a 15 años), centrales (de 16 a 60 años) y marginales adultos (61 o más años).

Como se dijo, en los productores agrícolas la actividad parcelaria es la que absorbe la mayor fracción de la capacidad de trabajo de las unidades económicas familiares. Es la actividad que concentra en mayor proporción a los trabajadores en todos los grupos de edad y sexo. En el caso 
de los varones de 8 a 15 años de edad, $82.6 \%$ de los que trabajan lo hacen exclusivamente en las labores de la tierra. Las niñas, además de participar principalmente en la parcela, se emplean en una mayor proporción que los niños en el negocio familiar (véanse los cuadros 5 y 6 ).

En el grupo de productores agrícolas, el trabajo infantil es el que menos se presenta bajo relaciones salariales. La participación en esta actividad es casi insignificante si se lleva a cabo como actividad única. Sin embargo, se convierte en la tercera en importancia, para este grupo de edad, cuando se combina con el trabajo parcelario.

Los trabajadores masculinos entre 16 y 60 años de edad, definidos como trabajadores centrales, están ocupados mayoritariamente en la parcela. Se destaca, además, la alta proporción de individuos que venden fuerza de trabajo como una actividad complementaria a las labores agrícolas dentro de la unidad.

Los hombres mayores de 60 años que trabajan presentan, en general, el mismo comportamiento que los trabajadores centrales masculinos, con la peculiaridad de que la proporción que se emplea exclusivamente en la parcela es mayor que en los centrales $(78.2$ y 61.3 por ciento, respectivamente; véase el cuadro 5). La actividad que sigue en importancia al trabajo exclusivo en la parcela es la combinación de éste con el trabajo asalariado.

Finaimente, en este grupo las mujeres mayores de 15 años se dedican principalmente a la actividad parcelaria; sin embargo, son las que presentan la proporción más alta de trabajadores que se ocupan exclusivamente en el negocio familiar en comparación a todos los grupos de edad y sexo (véase el cuadro 6).

Así, en el grupo de productores agrícolas pueden definirse las características de ocupación por una participación mayoritaria de mujeres, niños y hombres en la actividad parcelaria. Fara los varones mayores de 16 años, la segunda actividad en importancia es la venta de fuerza de trabajo, y para las mujeres de todas las edades tiene mayor relevancia el negocio familiar.

En el grupo de trabajadores por cuenta propia, la participación económica de los niños en diversas actividades constituye un elemento de gran importancia para la familia. El trabajo infantil se destina principalmente al negocio familiar $73.8 \%$ de las niñas y $51.7 \%$ de los niños que laboran se emplean en esta actividad). Otro rubro donde se concentran este tipo de trabajadores, especialmente varones, es en la parcela. No es frecuente que estos miembros combinen la venta de fuerza de trabajo con otras labores productivas; cabe señalar, sin embargo, que el porcentaje de los niños que trabajan únicamente por un salario es mayor que el observado en el otro grupo de productores independientes (véanse los cuadros 5 y 6).

En el negocio familiar se emplea $49.4 \%$ de los trabajadores centrales 


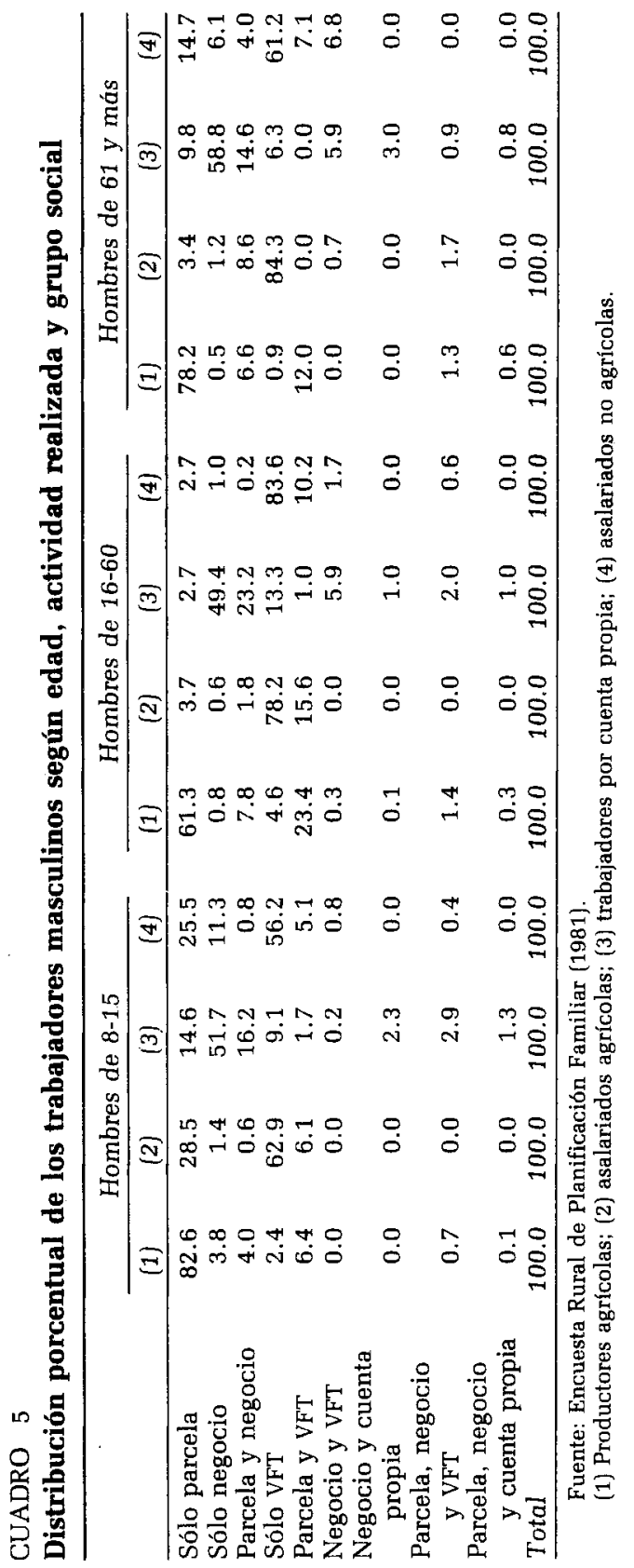




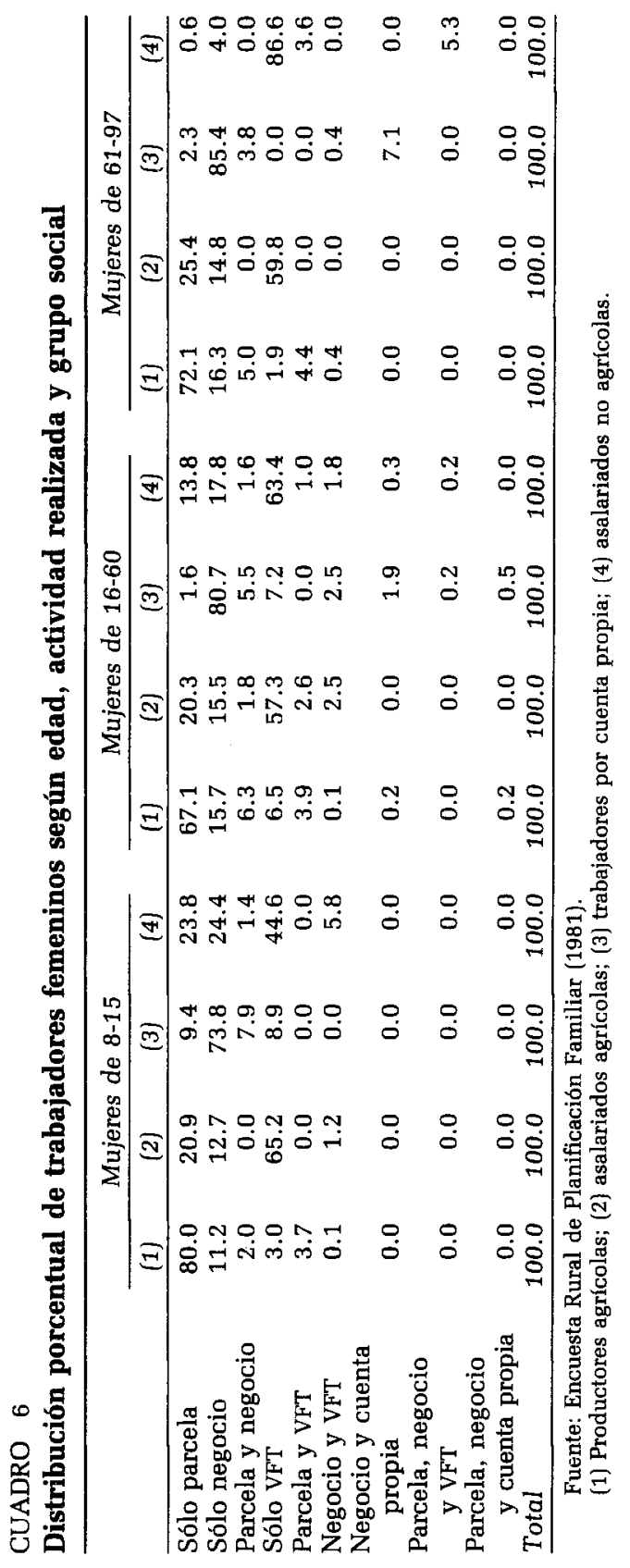


masculinos. Resalta el hecho de que esta proporción es menor que la que muestran los otros miembros. Una cuarta parte de los trabajadores masculinos entre 16 y 60 años, así como alrededor de $15 \%$ de los trabajadores de mayor edad, se dedican conjuntamente a realizar tareas en la parcela y el negocio familiar. Por otro lado, la proporción de personas que venden su fuerza de trabajo, sumando los que lo hacen de manera exclusiva o en combinación con otras actividades, es relativamente menor que la de los demás trabajadores de la misma edad en el otro grupo de productores independientes. Sin embargo, si se considera sólo la exclusiva venta de fuerza de trabajo, la situación se invierte.

En comparación con los otros grupos sociales, los trabajadores masculinos del grupo por cuenta propia diversifican más sus actividades. Esto puede deberse a que en la actividad principal de estos hogares se emplea a un gran número de mujeres, lo que facilita que los hombres realicen otras actividades (véase el cuadro 6). En el negocio familiar se concentra la participación económica de las mujeres de cualquier edad.

En el grupo de asalariados no agrícolas, los trabajadores infantiles presentan una gran diversificación de actividades; sin embargo, al igual que para los demás trabajadores asalariados, la venta de fuerza de trabajo constituye su principal actividad $(56.2 \%$ de los niños y $44.6 \%$ de las niñas que trabajan perciben un salario). Por otro lado, una cuarta parte de los trabajadores menores de 16 años se dedica exclusivamente a las labores de la parcela. Asimismo, un porcentaje importante de las niñas $(24.4 \%)$ se emplea en el negocio familiar, mientras que únicamente uno de cada diez varones menores de 16 años labora en esta actividad.

Los trabajadores centrales masculinos, en $83.6 \%$, se dedican exclusivamente a la venta de su fuerza de trabajo. Aun aquellos que realizan una actividad distinta no se desligan, generalmente, de la venta de trabajo, restando una mínima proporción de estos trabajadores (3.9\%) que sólo trabajan de manera independiente.

Tres quintas partes de los hombres mayores de 60 años que trabajan, únicamente venden su fuerza de trabajo. A diferencia de los trabajadores centrales, $14.7 \%$ se dedica exclusivamente a las labores parcelarias, lo que sumado al resto de estos trabajadores que desempeñan actividades sólo de manera independiente, da como resultado que una cuarta parte no venda su fuerza de trabajo. Esto puede deberse a que, por un lado, se trata de campesinos que han dejado de ser los jefes económicos del hogar, pero que siguen trabajando su parcela, mientras que otro familiar que se emplea como asalariado no agrícola, muy probablemente un hijo, aporta mayor ingreso para la manutención. Por otro lado, pueden ser personas no absorbidas por el mercado de trabajo, que buscan desarrollar alguna actividad económica de manera independiente en un negocio propio. Las trabajadoras, con excepción de las de mayor edad, presentan la mayor diversificación de actividades dentro del mismo grupo. 
Los miembros del grupo de asalariados agrícolas que realizan una actividad económica lo hacen mayoritariamente asalariándose. Cabe resaltar que las dos terceras partes de los niños que trabajan se asalarían. Las mujeres de 16 años o más se ocupan también principalmente en el trabajo asalariado y luego en la parcela. Por cierto, los porcentajes que presentan las mujeres trabajando exclusivamente en este recurso son muy superiores a los de los hombres de la misma edad (16 o más); los hombres centrales cuando trabajan en la parcela lo hacen combinando esta actividad con la venta de su fuerza de trabajo. Debemos subrayar que los trabajadores masculinos mayores de 60 años se dedican, si los comparamos con los asalariados no agrícolas, en mayor proporción a la venta exclusiva de fuerza de trabajo ( $84.3 \%$ frente a $61.2 \%$ ).

Se puede decir que la ocupación del jefe económico ejerce una influencia primordial en el desempeño de las actividades de los distintos miembros del hogar. Así, en las unidades campesinas una alta proporción de los trabajadores ejercen actividades parcelarias, mientras que para los miembros de los grupos de asalariados la mayor parte de los trabajadores se emplea en la venta exclusiva de su fuerza de trabajo. El grupo de trabajadores por cuenta propia muestra la misma tendencia aunque con la peculiaridad de que una proporción menor de los trabajadores centrales masculinos se emplea en la actividad primordial del hogar.

Además, se puede decir que la más elevada participación económica de los miembros del hogar en los grupos de productores agrícolas y trabajadores por cuenta propia está dada por la mayor participación de los miembros marginales, los cuales se ocupan principalmente en los recursos propios del hogar. Los trabajadores centrales masculinos en estos grupos, presentan mayor diversificación de actividades que el resto de los trabajadores, dada una superior participación en la combinación de la venta de fuerza de trabajo con la actividad parcelaria o bien con el negocio familiar.

En los grupos de asalariados, por el contrario, se tiende a diversificar más la actividad de los trabajadores marginales que la de los hombres de 16 años y más. La ocupación de los trabajadores marginales en estos grupos se dirige a la parcela y al negocio, por lo que se sostiene que esta participación se restringe a los hogares que cuentan con estos recursos. Lo anterior tiene lugar en $\mathbf{2 3 . 4 \%}$ de los hogares de los asalariados agrícolas y en $19.4 \%$ de los hogares de asalariados no agrícolas.

Para identificar si un mayor nivel de tecnificación desplaza la fuerza de trabajo familiar de las labores en la parcela, o si el uso de métodos más modernos de trabajo parcelario modifica el destino que se le da al empleo de los miembros de la familia, se creó un indicador de los niveles de tecnificación. Se partió del uso de maquinaria en alguna labor del cultivo y la presencia de riego en la superficie de labor (véase Montañez, 1982, p. 46). De esta manera, se clasificó a las unidades que usaban maquinaria y tenían riego como de alta tecnificación, a las que disponían de uno u 
otro de estos recursos, como de tecnificación media, y las que no contaban ni con riego ni maquinaria como de baja tecnificación.

De todas las unidades familiares agrícolas, $60.2 \%$ no cuenta con riego en su tierra ni utiliza maquinaria para la realización de las labores agrícolas, lo que las ubica dentro de lo que hemos denominado nivel de baja tecnificación. Estas unidades no han incorporado plenamente nueva tecnología, insumos y procesos de trabajo en sus explotaciones, lo que muy probablemente las coloca en difícil situación como productores de mercancías agrícolas; esto es, son unidades con desventajas competitivas que pueden dificultar su reproducción como unidades de producción.

Si bien en todas las unidades de productores agrícolas es muy elevada la proporción de integrantes del hogar que trabajan, los datos muestran una ligera tendencia a que dicha proporción disminuya a medida que se eleva el nivel de tecnificación. Así, mientras que en las unidades temporales y no mecanizadas realiza una actividad económica $60.3 \%$ de las personas de más de 7 años de edad, en las más tecnificadas dicho porcentaje es de $55.5 \%$ (véase el cuadro 7 ).

CUADRO 7

Porcentaje de integrantes del hogar que trabajan, según edad y sexo, por nivel de tecnificación de los productores agrícolas

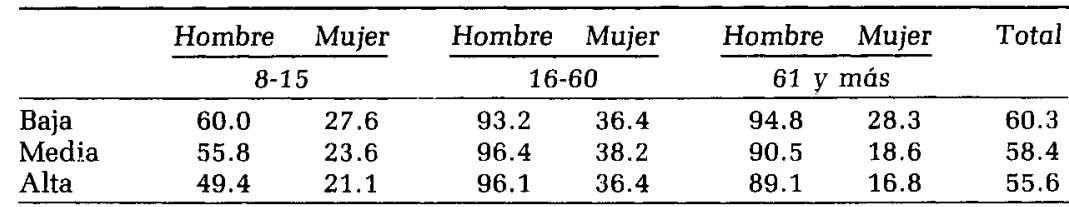

Fuente: Encuesta Rural de Planificación Familiar (1981).

Las diferencias más marcadas en cuanto al uso económico de la fuerza de trabajo marginal, según la calidad de los recursos productivos disponibles, se encuentra en el grupo de hombres menores de 16 años y en el de las mujeres mayores de 60 años. Mientras que en las unidades con más bajo nivel de tecnificación trabajan 60 y 28 por ciento de estos niños y mujeres, respectivamente, en las del nivel de alta tecnificación las cifras respectivas son 49.4 y 16.8 por ciento. Por el contrario, el porcentaje correspondiente a los trabajadores centrales no varía sustancialmente al cambiar las condiciones técnicas de la producción agrícola. Lo mismo sucede con las mujeres de 16 y 60 años, cuyo nivel de participación da cuenta de su importancia económica, independientemente de los recursos y métodos empleados en la producción agrícola. Asimismo, virtualmente la totalidad de los hombres de mayor edad participa en el trabajo, lo que nos 
indica que en los grupos agrícolas su participación es indispensable bajo cualquier condición técnica.

Con base en lo expuesto hasta ahora, pareciera que a una mayor tecnificación corresponde una menor participación en el trabajo de los niños y de las mujeres de mayor edad. Esto puede ser reflejo tanto de la reducción del trabajo necesario para la explotación de la parcela como de la creciente pérdida de control en la asignación de su fuerza de trabajo por parte de la unidad familiar, a consecuencia de las constantes transformaciones tecnológicas. También puede responder a condiciones de vida más favorables, resultado de mejoras en la eficiencia productiva de estas unidades, que facilitan que este tipo de personas puedan liberarse de las actividades económicas.

En cuanto a las tareas que realizan las personas que trabajan, se observan diferencias entre quienes laboran en la parcela y quienes venden su fuerza de trabajo en los grupos con niveles distintos de tecnificación. En el cuadro 8 se muestra que conforme se eleva el nivel de tecnificación decrece el número de trabajadores entre 16 y 60 años, tanto femeninos como masculinos, dedicados al trabajo parcelario; aumenta a la vez el de los que parcial o exclusivamente venden su fuerza de trabajo. Cabría senalar que el trabajo asalariado de cualquier modo, se presenta en todos los niveles de tecnificación en alrededor de $20 \%$ de los trabajadores centrales.

Entre los 16 y los 60 años de edad, $35.2 \%$ de los hombres y $18.8 \%$ de las mujeres pertenecientes a las unidades de producción más tecnificadas venden, ya sea como única actividad o combinada con otras, su fuerza de trabajo; por otro lado, estas cifras son, 28.0 y 10.8 por ciento, respectivamente, en las explotaciones temporaleras no mecanizadas. Estos datos indican que la mayor tecnificación de la producción facilita que las personas cuya fuerza de trabajo es central la ofrezcan en los mercados locales de trabajo. Éstos favorecen en general el empleo de ese tipo de trabajador. Asimismo, ser integrante de las unidades productivas más tecnificadas puede significar para el trabajador asalariado un recurso que permite su subsistencia en épocas de desempleo, por lo que las fluctuaciones del mercado de trabajo no conducen necesariamente a la migración de este tipo de trabajadores. Sin embargo, y a manera de hipótesis, lo anterior no significa que la tendencia a la proletarización sea menor en el caso de las unidades de producción dotadas con recursos de menor calidad; por el contrario, al no poder reproducirse en el marco de un estrecho e irregular mercado local de trabajo, estas unidades pueden expulsar un número importante de sus miembros bajo la forma de migración. 


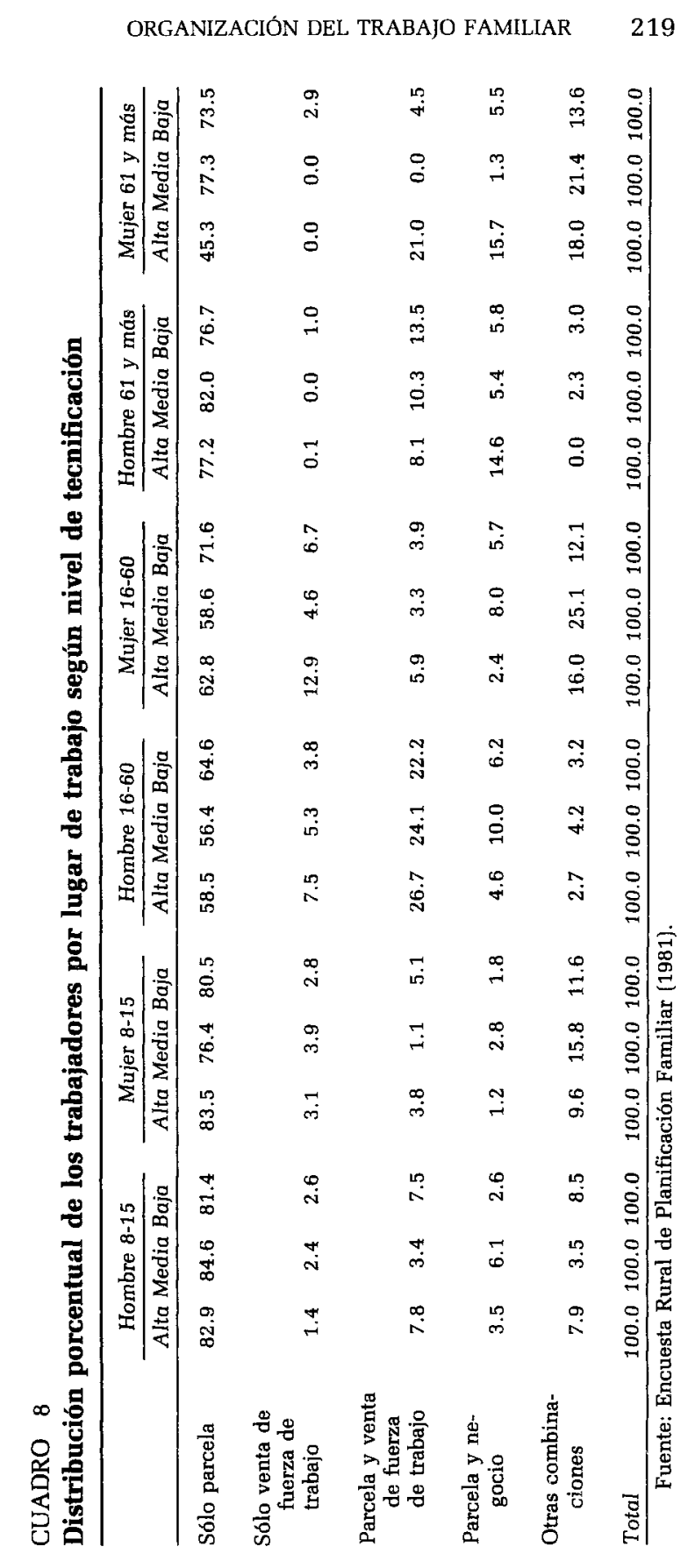




\section{Algunas consideraciones generales acerca de la fecundidad de los grupos sociales analizados}

Pasaremos ahora a exponer algunos datos que nos permitan una primera aproximación al análisis de la conducta reproductiva de los grupos sociales hasta aquí presentados. Debemos señalar que el hecho de que se esté considerando a personas que residen en localidades menores a 2500 habitantes, poblaciones dispersas y, en la mayoría de los casos, aisladas de los centros urbanos y de los servicios que ahi se tienen, puede hacer más lentas las transformaciones ideológicas y sociales (que influyen en los cambios de la conducta reproductiva) que ocurren conforme se desarrolla el capitalismo. Esto se debe, en parte, al mismo aislamiento sociocultural, así como a la permanencia de formas tradicionales de vida que dependen de procesos de producción no típicamente capitalistas, como son la producción basada en el trabajo no retribuido de los miembros de la familia y la existencia de normas de consumo restringidas a la producción de la unidad económica familiar.

Sin embargo, la presencia de distintas condiciones económicas para la reproducción cotidiana y biológica puede dar lugar a que los grupos sociales presenten diferencias en su conducta reproductiva. El principal elemento de la base económica que debemos considerar es el lugar que ocupan los individuos en las relaciones sociales de producción. Así, es posible distinguirlos a partir de su propiedad o no de recursos económicos materiales. Tenemos, por un lado, a los desposeídos de estos recursos, en los que la actividad económica principal de los hogares se realiza bajo una forma típicamente capitalista, a saber, vendiendo la fuerza de trabajo por un salario. Por otro lado están los que sí poseen esos recursos y que, por tanto, tienen la posibilidad de desarrollar una actividad de manera independiente, como es el caso de los trabajadores por cuenta propia y los productores agrícolas, quienes, por lo general, constituyen unidades económicas familiares.

Puede plantearse la hipótesis de que la conducta reproductiva de los grupos donde existen estas unidades económicas está caracterizada por una alta fecundidad, asociada al funcionamiento histórico de la organización económica de estas unidades. Es de esperar que las transformaciones en los procesos de producción, así como el mejoramiento en las condiciones de vida y salud, den lugar a condiciones distintas para la reproducción que favorezcan niveles menores de fecundidad. Si se considera, en este sentido, el número promedio de hijos nacidos vivos de las mujeres unidas al final de su vida reproductiva (aquéllas entre 45 y 49 años), se observa que el nivel de fecundidad de las mujeres de los grupos de trabajadores independientes es más elevado que el de las pertenecientes a los grupos de asalariados (véase el cuadro 9).

Sin embargo, conforme se analizan cohortes más jóvenes, se puede 
CUADRO $\mathbf{9}$

Número promedio de hijos nacidos vivos de mujeres unidas según cuatro grupos de edad y grupo social

\begin{tabular}{lcccc}
\hline $\begin{array}{l}\text { Grupos de } \\
\text { edad }\end{array}$ & $\begin{array}{c}\text { Productores } \\
\text { agrícolas }\end{array}$ & $\begin{array}{c}\text { Asalariados } \\
\text { agrícolas }\end{array}$ & $\begin{array}{c}\text { Cuenta } \\
\text { propia }\end{array}$ & $\begin{array}{c}\text { Asalariados no } \\
\text { agrícolas }\end{array}$ \\
\hline $30-34$ & 5.5 & 5.3 & 4.8 & 4.8 \\
$35-39$ & 7.3 & 6.9 & 6.7 & 6.6 \\
$40-44$ & 8.0 & 7.0 & 7.4 & 7.5 \\
$45-49$ & 8.4 & 7.7 & 8.8 & 7.3 \\
\hline
\end{tabular}

Fuente: Encuesta Rural de Planificación Familiar (1981).

ver que son las mujeres de los grupos de trabajadores no agrícolas quienes presentan un menor número de hijos nacidos vivos, comparándolos con los productores y asalariados agrícolas. De lo anterior podría esperarse que el grupo de trabajadores por cuenta propia muestre tamaños de familia menores en el futuro.

Al estudiar otro indicador de los niveles de fecundidad, la tasa global de fecundidad marital (TGF), podemos corroborar lo anterior. Este indicador señala que si se sigue el comportamiento reproductivo observado en el momento de la entrevista, las mujeres unidas del grupo de trabajadores por cuenta propia llegarían al final de su vida reproductiva con un número menor de hijos nacidos vivos (7.64 en la TGF marital contra 8.8, que es el dato al considerar el promedio de hijos nacidos vivos), siendo aún muy alta su fecundidad. Por el contrario, el otro grupo de productores independientes sigue presentando niveles de fecundidad muy altos en su tasa global de fecundidad (8.85; véase cuadro 10 ).

CUADRO 10

Tasas específicas de fecundidad marital de los diferentes grupos sociales

\begin{tabular}{|c|c|c|c|c|c|}
\hline $\begin{array}{l}\text { Grupos de } \\
\text { edad }\end{array}$ & $\begin{array}{l}\text { Productores } \\
\text { agrícolas }\end{array}$ & $\begin{array}{l}\text { Asalariados } \\
\text { agrícolas }\end{array}$ & $\begin{array}{l}\text { Cuenta } \\
\text { propia }\end{array}$ & $\begin{array}{c}\text { Asalariados no } \\
\text { agrícolas }\end{array}$ & Total \\
\hline $15-19$ & .424 & .446 & .407 & .424 & .427 \\
\hline $20-24$ & .398 & .458 & .438 & .382 & .407 \\
\hline $25-29$ & .295 & .308 & .213 & .298 & .291 \\
\hline $30-34$ & .293 & .226 & .240 & .179 & .250 \\
\hline $35-39$ & .224 & .219 & .213 & .143 & .205 \\
\hline $40-44$ & .112 & . 120 & .014 & .090 & .099 \\
\hline $45-49$ & .011 & .017 & .003 & .006 & .011 \\
\hline $\begin{array}{l}\text { Tasa global de } \\
\text { fecundidad } \\
\text { marital }\end{array}$ & 8.85 & 8.97 & 7.64 & 7.61 & 8.44 \\
\hline
\end{tabular}

Fuente: Encuesta Rural de Planificación Familiar (1981). 
La diferencia en las TGF maritales entre los grupos de trabajadores independientes nos lleva a plantear que, si bien en ambos grupos existe una alta participación económica de los miembros del hogar, el significado e importancia de ésta puede ser distinto, lo que podría explicar los niveles de fecundidad que se observan. Sobre este punto se puede señalar, en primer lugar, que es relevante el tipo de recurso material que se posea, lo que puede asociarse, entre otras cosas, con el tipo de trabajador que se necesita para su funcionamiento, pudiendo ser que el negocio sea más flexible respecto a quién participa y durante cuánto tiempo. El que haya en el negocio familiar una menor participación de niños y una mayor proporción de mujeres desempeñando una actividad económica, puede favorecer menores niveles de fecundidad en el grupo de trabajadores por cuenta propia.

Podemos suponer, por otro lado, que este grupo social tiene mayor posibilidad de un contacto social cotidiano más allá del ámbito familiar que el grupo de productores agrícolas, debido al tipo de actividad que desarrolla, ligado con más fuerza al mercado capitalista. El que el consumo familiar del grupo de trabajadores por cuenta propia deba realizarse necesariamente por medio de un intercambio mercantil determina la presencia de un patrón caracterizado por la creciente incorporación de nuevos bienes; entre los productores agrícolas la norma de consumo todavía está muy ligada a la producción de la unidad económica familiar. La creación de nuevas necesidades repercute sobre las expectativas de vida de la familia, por ejemplo, en términos de la educación de los hijos; al mismo tiempo, la realización de estas expectativas se ve obstaculizada por un tamaño muy grande de familia, dadas las restricciones en el ingreso.

En general, es posible que los integrantes del grupo de los trabajadores por cuenta propia se estén integrando más a la ideología burguesa que propone tamaños de familia más pequeños y tenga mayores posibilidades de conocer y adoptar programas de planificación familiar. Esto da como resultado una alta proporción de mujeres unidas que regulan su fecundidad mediante el uso de métodos anticonceptivos ( $37.3 \%$ ), como se puede observar en el cuadro 11, en contraste con las mujeres unidas del grupo de productores agrícolas, donde sólo $22.5 \%$ son usuarias de métodos.

En relación con el grupo de productores agrícolas, diferenciado según las características técnicas de las unidades, observamos que las que utilizan riego y maquinaria en la explotación agrícola tienen una TGF marital menor que la del resto de los productores (véase el cuadro 12). Esto puede asociarse a que, si bien se sigue utilizando trabajo familiar en las labores parcelarias, el uso de maquinaria hace que éste sea menos intensivo a lo largo de todo el ciclo de cultivos, aunque en los momentos críticos se puede necesitar un alto número de trabajadores, por lo que la unidad se ve forzada a contratarlos.

Además, el hecho de incorporar nuevos procesos de trabajo hace que 
CUADRO 11

Proporción de mujeres unidas que usan anticonceptivos según su edad en los diferentes grupos sociales

\begin{tabular}{lcccc}
\hline $\begin{array}{l}\text { Grupos de } \\
\text { edad }\end{array}$ & $\begin{array}{c}\text { Productores } \\
\text { agrícolas }\end{array}$ & $\begin{array}{c}\text { Asalariados } \\
\text { agrícolas }\end{array}$ & $\begin{array}{c}\text { Cuenta } \\
\text { propia }\end{array}$ & $\begin{array}{c}\text { Asalariados no } \\
\text { agrícolas }\end{array}$ \\
\hline $15-19$ & 13.1 & 6.6 & 17.5 & 15.9 \\
$20-24$ & 29.3 & 19.5 & 39.8 & 33.3 \\
$25-29$ & 29.2 & 33.8 & 40.3 & 43.5 \\
$30-34$ & 24.3 & 31.5 & 50.3 & 43.4 \\
$35-39$ & 28.7 & 29.5 & 39.1 & 43.5 \\
$40-44$ & 22.4 & 17.7 & 32.5 & 36.5 \\
$45-49$ & 8.0 & 8.8 & 20.4 & 14.0 \\
Total & 22.5 & 23.1 & 37.3 & 35.8 \\
\hline
\end{tabular}

Fuente: Encuesta Rural de Planificación Familiar (1981).

CUADRO 12

Tasas específicas de fecundidad marital por características técnicas

\begin{tabular}{|c|c|c|c|}
\hline $\begin{array}{l}\text { Grupos de } \\
\text { edad }\end{array}$ & $\begin{array}{c}\text { Característica } \\
\text { técnica } \\
1 \\
\text { (Alta) } \\
\end{array}$ & $\begin{array}{c}\text { Característica } \\
\text { técnica } \\
2 \\
\text { (Media) } \\
\end{array}$ & $\begin{array}{c}\text { Característica } \\
\text { técnica } \\
3 \\
\text { (Baja) }\end{array}$ \\
\hline $15-19$ & .554 & .393 & .430 \\
\hline $20-24$ & .486 & .427 & .384 \\
\hline $25-29$ & .260 & .313 & .294 \\
\hline $30-34$ & .141 & .258 & .333 \\
\hline $35-39$ & .061 & .177 & .266 \\
\hline $40-44$ & .081 & .122 & .133 \\
\hline $45-49$ & .029 & .014 & .008 \\
\hline $\begin{array}{l}\text { Tasa global de fecun- } \\
\text { didad marital }\end{array}$ & 8.06 & 8.52 & 9.14 \\
\hline
\end{tabular}

Fuente: Encuesta Rural de Planificación Familiar (1981).

estos productores agrícolas tengan una mayor dependencia del dinero para lograr su reproducción; esta relación con el mercado capitalista puede redundar en un cambio en sus expectativas de vida que influya en su conducta reproductiva y los lleve a tener un número menor de hijos. De hecho, la proporción de mujeres unidas que utilizan métodos anticonceptivos es de $35.6 \%$ en los productores con alta tecnificación, prácticamente igual a lo que se observa para las mujeres del grupo de asalariado no agrícola, mientras en el grupo de baja tecnificación es de $16.3 \%$ (cuadro 13).

Restan por analizar los grupos en los que existe una menor presencia de unidades familiares y que tienen un alto nivel de dependencia del mercado tanto para emplearse como para satisfacer el consumo familiar, como es el caso de los trabajadores asalariados. Podemos plantear que en 
CUADRO 13

Proporción de mujeres unidas que usan anticonceptivos según su edad en las diferentes características técnicas (porcentajes)

\begin{tabular}{|c|c|c|c|}
\hline $\begin{array}{l}\text { Grupos de } \\
\text { edad }\end{array}$ & $\begin{array}{c}\text { Característica } \\
\text { técnica } \\
1 \\
\text { (Alta) } \\
\end{array}$ & $\begin{array}{c}\text { Caracteristica } \\
\text { técnica } \\
2 \\
\text { (Media) } \\
\end{array}$ & $\begin{array}{c}\text { Característica } \\
\text { técnica } \\
3 \\
(\text { Baja) } \\
\end{array}$ \\
\hline $15-19$ & 15.8 & 9.9 & 14.6 \\
\hline $20-24$ & 43.3 & 32.0 & 15.3 \\
\hline $25-29$ & 44.9 & 39.6 & 20.1 \\
\hline $30-34$ & 41.2 & 40.5 & 14.0 \\
\hline $35-39$ & 40.5 & 38.4 & 23.5 \\
\hline $40-44$ & 31.5 & 30.0 & 15.1 \\
\hline $45-49$ & 10.6 & 11.5 & 5.5 \\
\hline Total & 35.6 & 30.5 & 16.3 \\
\hline
\end{tabular}

Fuente: Encuesta Rural de Planificación Familiar (1981).

la medida en que se trata de unidades familiares donde se encuentran separadas las esferas de la producción y la reproducción, esto es, donde las posibilidades de reproducción como trabajadores de los miembros del hogar están fuera del control del núcleo familiar y dependen de un elemento externo a ella (las condiciones del mercado de fuerza de trabajo), se observarán niveles de fecundidad menores que los de los grupos de trabajadores independientes. Esto es cierto para los asalariados no agrícolas, pero no para los asalariados agrícolas, los que muestran una conducta semejante a la de los productores agrícolas. Este fenómeno podría estar relacionado con el hecho de que este grupo mantiene importantes lazos, para su reproducción, con economías campesinas; también puede influir el que prevalezcan entre ellos mayores niveles de mortalidad y que el tipo de actividad que realizan los relacione a formas de vida y pensamiento tradicionales que hacen que su conducta reproductiva se caracterice por altos niveles de fecundidad y una baja proporción de mujeres unidas que usan anticonceptivos (véanse los cuadros 10 y 11).

En la agricultura, como es sabido, los procesos de trabajo no sufren, en general, transformaciones tan aceleradas como otras ramas de la economía capitalista; esto tiene una estrecha relación con el nivel de calificación de los trabajadores. Así, se puede suponer que los mayores niveles de calificación de los asalariados no agrícolas (que implican, en principio, mayores niveles de escolaridad), se asocian a conductas reproductivas más modernas. Debemos considerar además que no sólo el mismo tipo de trabajo que se desarrolla en la agricultura es distinto al de otras ramas en términos de la organización del trabajo (por ejemplo, la forma de cooperación), sino que el propio salario presenta diferencias: mientras que el salario agrícola, por la eventualidad general del empleo en la agricul- 
tura, es individual, el salario no agrícola es lo que podríamos denominar "familiar", diseñado para un tamaño pequeño de familia. La presencia de salarios individuales hace que los asalariados agrícolas necesiten cierto número de ellos para conformar un ingreso familiar suficiente para su reproducción. Esto se logra, en un mercado de fuerza de trabajo con demanda eventual, sólo mediante la oferta de un amplio número de trabajadores, intentando asegurar así la contratación de algunos.

Esto podría explicar los altos niveles de fecundidad en este grupo. Si bien no presentan niveles de uso de la fuerza de trabajo familiar como los grupos independientes, la participación económica de los hombres es muy superior a la del otro grupo de asalariados. Además, podemos suponer que las características de los mercados de fuerza de trabajo locales están provocando un amplio proceso migratorio en este grupo, que debemos analizar para comprender mejor su conducta reproductiva.

Podemos concluir que no existe una relación directa entre la pertenencia a unidades económicas familiares, donde se utiliza en grandes proporciones la fuerza de trabajo familiar, y la existencia de conductas reproductivas caracterizadas por altos niveles de fecundidad. Los procesos económicos como la tecnificación de la agricultura y la monetarización de las transacciones en el medio rural, que serían ejemplificados en nuestros grupos de productores agrícolas de alta tecnificación y los trabajadores por cuenta propia, son elementos que nos ayudan a comprender por qué grupos que funcionan como unidades domésticas de producción, que históricamente han presentado una alta fecundidad, muestran una conducta reproductiva distinta.

\section{Bibliografía}

Bartra, Armando, La explotación del trabajo campesino por el capital, Macehual, México, 1979, pp. 85 y ss.

CEPAL, Economía campesina y agricultura empresarial, Siglo xXI, México, 1982, pp. 104-109.

IMSS-JSPF, Encuesta rural de planificación familiar, Documento metodológico, IMSS, México, 1982

Margulis, Mario, Contradicciones en la estructura agraria y transferencias de valor, El Colegio de México, México, 1979, p. 2.

Martínez, Marielle y Teresa Rendón, "Fuerza de trabajo y reproducción campesina", en Comercio Exterior, vol. 8, núm. 6, 1978, pp. 665-666.

, "Algunas estrategias de reproducción de las unidades domésticas campesinas", en Investigación Demográfica en México - 1980, CONACYT, México, 1982, pp. 601-603.

Montan̄ez, Carlos, El cultivo de maíz en México, Centro de Ecodesarrollo, México, 1982 , p. 46.

Warman, Arturo, Y venimos a contradecir, Ed. de la Casa Chata, México, 1976, pp. 310-312. 
